\title{
Informações on-line sobre transporte intra-hospitalar de pacientes críticos adultos*
}

\author{
Online information about Intrahospital Transport of Adults Patients Critical \\ Información en línea sobre el transporte intrahospitalario \\ de los pacientes enfermos graves adultos
}

Valnice de Oliveira Nogueira ${ }^{1}$, Heimar de Fátima Marin², Isabel Cristina Kowal Olm Cunha ${ }^{3}$

\begin{abstract}
RESUMO
Objetivo Este artigo relata o desenvolvimento de um Web site educacional em transporte intra-hospitalar de pacientes críticos adultos. Métodos: É uma pesquisa aplicada que visa oferecer informações para profissionais de saúde, discentes e docentes. O método de desenvolvimento utilizado, foi o de Trochim. Resultados: compreendeu 4 etapas: conceituação, desenvolvimento, implementação e a avaliação numa forma interativa e cíclica. Conclusões: Sugere recomendações para a prática clínica baseada em evidências, na busca da melhor qualidade da assistência. Estas informações podem ser acessadas na World Wide Web, na URL: www.unifesp.br/ denf/nien/transporte.
\end{abstract}

Descritores: Transporte de pacientes; Cuidados críticos; Medicina baseada em evidências

\begin{abstract}
Objective: This study describes the development of an educational web site about intrahospital transportation of critically ill patients. Methods: The goal of this Web site is to provide information to health care professionals, students, and faculty members about safe transportation of this type of patient. Trochim's model for development of Web sites served as a conceptual framework for this study. Results: The model consists of four general phases: conceptualization, development, implementation, and evaluation. These phases are interrelated to each other and constitute a recurring cycle. Conclusions: The newly developed web site may serve as a practical guideline to improve the quality of care when transporting critical ill patients. It can be accessed in the World Wide Web at URL: www.unifesp.br/denf/nien/transporte.
\end{abstract}

Keywords: Transportation of patients; Critical care; Evidence-based medicine

\section{RESUMEN}

Objetivo En este artículo se relata el desarrollo de un Web site educativo en relación al transporte intra hospitalario de pacientes críticos adultos. Métodos: Se trata de una investigación aplicada que visa ofrecer informaciones a profesionales de salud, discentes y docentes. El método de desarrollo utilizado, fue el de Trochim. Resultados: comprendió 4 etapas: conceptuación, desarrollo, implementación y la evaluación de forma interactiva y cíclica. Conclusiones: Se sugiere recomendaciones para la práctica clínica basada en evidencias, a fin de mejorar la calidad de la asistencia. Estas informaciones pueden ser buscadas en la World Wide Web, en la URL: www.unifesp.br/denf/nien/transporte .

Descriptores: Transporte intra hospitalario pacientes críticos; Protocolo de conductas

\footnotetext{
* Trabalho extraído da Dissertação de Mestrado apresentada à Universidade Federal de São Paulo - UNIFESP - São Paulo(SP), Brasil, em 2003.

1 Mestre em Enfermagem. Professora do Curso de Enfermagem do Centro Universitário Nove de Julho - São Paulo (SP), Brasil..

2 Doutora em Ciências. Professora Associada Livre-Docente do Departamento de Enfermagem da Universidade Federal de São Paulo - UNIFESP - São Paulo (SP), Brasil.

3 Doutora em Saúde Pública. Professora Adjunta do Departamento de Enfermagem da Universidade Federal de São Paulo - UNIFESP - São Paulo(SP), Brasil.
} 


\section{INTRODUÇÃO}

Nas áreas das ciências sociais, econômicas, biomédicas e exatas em âmbito mundial, a informática permitiu uma série de avanços. As megatendências ocasionam mudanças nas esferas sociais, políticas e tecnológicas e influenciam a sociedade como um todo. A era da informática, que é classificada como uma das megatendências, caminha com a globalização em prol da qualidade de vida, da formação de profissionais e da utilização de serviços em geral.

A informatização é um recurso que estabelece a ligação entre o mundo real e o virtual, modificando, significativamente, as formas de pensamento, ação, trabalho e convívio de um indivíduo com a sociedade e as organizações, tudo isto graças à tecnologia computacional.

Com a evolução dos computadores e dos recursos da informática, surgiu a internet que também ocupa um importante papel na evolução da tecnologia. Trata-se da rede mundial de computadores, que permite a conexão de um computador localizado em uma parte do mundo a um outro qualquer, próximo ou distante fisicamente. É um grande veículo de informações que possibilita aquisição de conhecimento, resolução de dúvidas e, até mesmo, proporciona lazer e relações interpessoais em tempo real.

De modo geral, os recursos da internet hoje disponíveis, ou mesmo da informática, possibilitam a compilação, arquivamento e distribuição de informações, proporcionando a criação de ações educativas, administrativas e de assessoria nas diversas áreas de concentração, sendo tais informações amplamente compartilhadas através da internet, sem restrição de data, hora, distância e limite de participantes. A utilização destas ferramentas em ações de cunho educativo, especificamente em programas de educação à distância, é um caminho alternativo viável e passível de execução, já utilizado por várias universidades e institutos de ensino no Brasil e exterior.

Educação à distância (EAD) é definida como

o processo de ensino-aprendizagem, mediado por tecnologias, onde professores e alunos estão separados espacial e/ou temporalmente. No ensino à distância, a ênfase é dada ao papel do professor (como alguém que ensina à distância) ${ }^{(1)}$.

O ensino à distância é fundamental no desenvolvimento da educação, sendo visto como a nova estratégia para o século XXI que possibilitará a oportunidade e o acesso igualitário à informação. Assim, educadores e educandos deverão desenvolver habilidades e adquirir conhecimentos para as inovações advindas desta ferramenta( ${ }^{(2)}$.
Diogo $^{(3)}$ encara a EAD como mais um recurso a ser usado pela educação continuada para o desenvolvimento de programas de treinamento e reciclagem para a equipe de enfermagem, sempre que se fizer necessário. A enfermagem deverá aproveitar esta nova estratégia de ensino, visto que as possibilidades de manuseio deste método estão lançadas e a contemporização da nova forma de educar torna-se inevitável e imprescindível.

A informação é o elemento essencial para a ação com qualidade, mas para que seja útil e recuperável, deve estar contida em base de dados onde seja cadastrada e compartilhada com os profissionais de saúde, em prol das ações de enfermagem direcionadas ao indivíduo, família e comunidade ${ }^{(4)}$.

A enfermagem, como tantas outras profissões da área da saúde, é dependente da informação. $\mathrm{O}$ volume de dados utilizados por enfermeiros tem aumentado cada vez mais, de acordo com a evolução alcançada nas áreas da ciência e tecnologia. Entretanto, vale enfatizar que em termos de volume de dados e informações cadastradas e manipuladas no dia-a-dia, as unidades de Emergência e Terapia Intensiva são as que mais se destacam.

Nestes setores encontram-se freqüentemente indivíduos portadores de afecções classificadas de pequena a alta complexidade e, por esta razão, os últimos citados requerem intervenções imediatas e precisas. Estas intervenções, em algumas ocasiões devem ser realizadas fora da unidade de origem dos pacientes, e o transporte intrahospitalar torna-se parte integrante deste processo.

Por definição, o transporte intra-hospitalar é o encaminhamento temporário ou definitivo de pacientes por profissionais de saúde dentro do ambiente hospitalar, seja para fins diagnósticos ou terapêuticos.

Este procedimento, por ocorrer comumente nas unidades de atendimento supracitadas, é realizado de forma automática, sendo freqüentemente não valorizado, pelo fato de não transpor o cliente fora dos limites físicos do ambiente hospitalar e ter a segurança da compensação clínica imediata, se houver intercorrência no percurso. Outro fator contribuinte para a não valorização seria a não-utilização ou sub utilização de materiais e equipamentos para efetuar o procedimento. Vale destacar, que esta atitude é contra a ética, a moral e o dever do profissional da saúde que não valoriza o ato de transportar pacientes gravemente enfermos, em nível intra-hospitalar.

O treinamento e aperfeiçoamento constante dos profissionais envolvidos nesse procedimento, assim como a padronização de ações e equipamentos e, ainda, a garantia das perfeitas condições de uso e a revisão dos materiais necessários ao transporte, são fatores importantes para o sucesso desta atividade, que deve assegurar todo o benefício e segurança ao paciente que é transportado. 
Considerando os avanços que se processam no setor saúde em favor das inovações tecnológicas, pelo exercício das atividades profissionais em unidades consideradas de alta complexidade experienciando as deficiências na prática diária, os objetivos deste estudo foram: desenvolver uma página educacional na World Wide Web, disponibilizando e representando informações pertinentes à educação continuada,em transporte intra-hospitalar de pacientes críticos; avaliar o conteúdo destas informações com especialistas na área de informática, de emergência, de cuidados intensivos e com enfermeiros generalistas; propor um protocolo de condutas para a realização de um transporte intra-hospitalar de pacientes críticos adultos.

\section{MÉTODOS}

Este estudo constituiu-se numa pesquisa aplicada inserida na Linha de Pesquisa de Informática em Saúde do Núcleo de Informática em Enfermagem da Universidade Federal de São Paulo (NIEn - Departamento de Enfermagem - UNIFESP - EPM).

Para construir um website, utilizou-se uma vasta série de aplicativos e recursos de informática. A metodologia de desenvolvimento seguiu as recomendações de Trochim ${ }^{(5)}$, que propôs um modelo de criação de websites, baseado em uma estrutura para definir quatro etapas de forma interativa e cíclica de avaliação, que mescla atividades de desenvolvimento, planejamento e avaliação.

- A primeira fase foi a de conceituação, ou seja, de organização e apresentação dos conteúdos. As informações foram coletadas por meio de um levantamento bibliográfico sobre o tema nas seguintes bases de dados literários: Medline -Medical Literature Analysis and Retrieval System On Line, Lilacs - Literatura Latino Americana e do Caribe em Ciências da Saúde, PubMed National Library of Medicine e SiBi -Sistema Integrado de Bibliotecas da Universidade de São Paulo.

- A segunda fase foi a de desenvolvimento, em que o conteúdo foi traduzido por intermédio dos recursos tecnológicos (software, linguagem, estrutura, gráficos e imagens). Utilizou-se o software Front Page da Microsoft ${ }^{\circledR}$ com Linguagem em HTML para editoração e o software Adobe Photoshop foi usado no tratamento das imagens disponibilizadas.

- A terceira fase foi a de implementação, na qual foram realizados os testes iniciais de navegação e utilização. A primeira atividade foi a disponibilização do website em um provedor gratuito e, posteriormente, na rede da Universidade Federal de São Paulo, Núcleo de Informática em Enfermagem (NIEn/ UNIFESP).

- A quarta fase foi a de avaliação, onde foram examinadas as operações e os efeitos durante um cur- to, médio ou longo período de tempo. Foram convidados a participar especialistas da área de cuidados intensivos, emergência, informática, e enfermeiros generalistas que concluíram a graduação no segundo semestre de 2002. Os encontros foram agendados previamente e, na ocasião da avaliação, os participantes assinaram o Termo de Consentimento Livre e Esclarecido e preencheram um questionário de avaliação. Os questionários de avaliação foram construídos, tomando como referência o instrumento de coleta de dados elaborado por Motta ${ }^{(6)}$ ao desenvolver e validar um software educacional de enfermagem e puericultura.

\section{RESULTADOS E DISCUSSÃO}

Para melhor compreensão dos resultados, dividiu-se em três subseções:

O site

O site é composto de 42 páginas, sendo 34 textuais e oito contendo 22 imagens distribuídas como ilustração para apoio didático. Das páginas textuais, oito são consideradas norteadoras, pois são as que compõem a opção de menu. Pode ser acessado pela intranet da UNIFESP diretamente interligado ao Núcleo de Informática em Enfermagem no endereço: http://www.unifesp.br/ denf/NIEn/transporte.

Ao acessar o endereço fornecido, o usuário encontra a página inicial do Website que o conduzirá às demais informações sobre transporte do paciente intra-hospitalar (Figura 1)

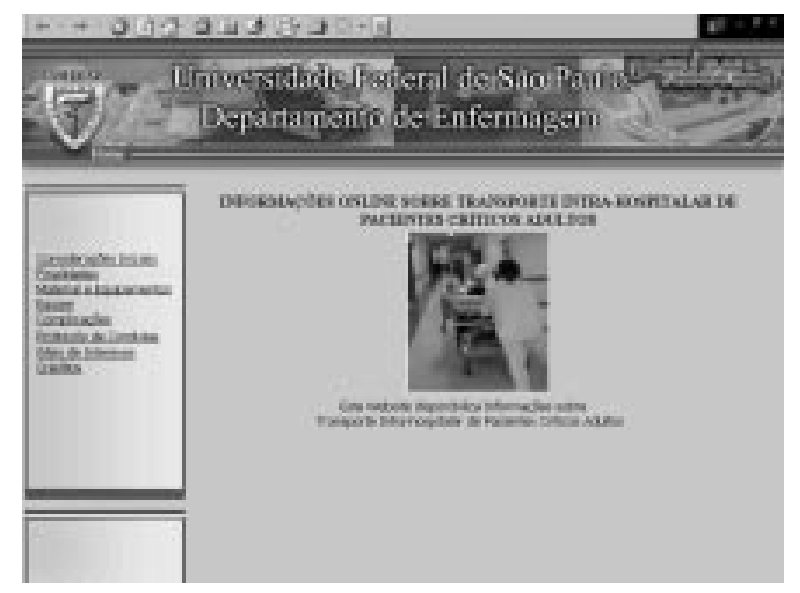

FIGURA 1 - Página inicial do website de transporte Intrahospitalar de Pacientes Críticos Adultos, SP, 2003.

As opções de menu seguem uma ordem didática para facilitar o entendimento das informações dispostas ao usuário.

A primeira e a segunda opção de menu reportam-se a conceituação, contendo portanto, as Considerações 
Iniciais e as Finalidades do website, respectivamente. A terceira opção intitulada Material e Equipamentos discute a indicação e a utilização de todos os materiais e equipamentos necessários ao transporte assim como possibilita a visualização dos mesmos. A opção de menu Equipe trata da formação profissional da equipe e do contingente de pessoal necessário ao transporte. Neste local também existe uma sessão de fotos e um vídeo onde há uma simulação de um transporte intrahospitalar.

A opção de menu Complicações, como o próprio nome diz, discute as principais intercorrências que podem acontecer no procedimento, e ainda apresenta sugestões para que se possa evitar ou abolir inconvenientes. A opção de menu Protocolo de Condutas sugere algumas ações a serem desenvolvidas antes, durante e após o procedimento, e são discutidas no próximo item. A sétima opção de menu intitulada Sites de Interesse estabelece links com entidades de classe, de ensino e pesquisa relacionadas especificamente ao profissional enfermeiro. Na última opção de menu, Créditos, obtém-se informações sobre a origem do material, os profissionais envolvidos e endereço eletrônico para entrar em contato com a autora.

No que diz respeito ao conteúdo das informações contidas no website, ressalta-se que este material foi preparado, tentando manter a clareza e a objetividade para facilitar o entendimento e promover o interesse.

$\mathrm{Na}$ área de pesquisa, em geral as páginas de internet são basicamente textuais e bem aceitas. Entretanto, é necessário entender que o excesso de texto pode ser encarado como um fator de distanciamento do usuário ao site. A autora ${ }^{(7)}$ discute, ainda, que o usuário deve obter uma informação navegando o menos possível, ou seja, evitando etapas desnecessárias. Sites que demoram muito para serem carregados, muitas vezes levam as pessoas a desistir da consulta. Enfatiza que um website deve despertar o interesse dos usuários para o assunto que apresenta.

Os recursos gráficos aliados à parte textual em um website são considerados peças fundamentais na retenção e incorporação da informação que gera o conhecimento. As situações de ensino-aprendizagem, que são complementadas com base nas representações de imagens, terão resultados satisfatórios.

É necessário elucidar que este website seguiu as determinações preconizadas pela Agência de Pesquisa e Qualidade no Cuidado à Saúde ${ }^{(8)}$ e o Código de Conduta de Saúde em Websites ${ }^{(9)}$. Estas instituições têm se preocupado com a qualidade do conteúdo e a fidedignidade da informação disposta na web, com os links estabelecidos pelo site, sua acessibilidade e navegação além da disposição de cores, letras e imagens. As determinações sugeridas pela Resolução COFEN
274/2002(10) , que dispõe sobre a utilização da Internet pelos Profissionais de Enfermagem, também foram respeitadas.

\section{AAVALIAÇÃO}

A avaliação para alguns autores ${ }^{(11)}$

é um processo dinâmico, por meio do qual se pode medir e comparar fatos, situações, realidades, procedimentos e serviços, de modo a nortear a tomada de decisão, os questionamentos da eficiência e sobretudo de eficácia dos programas.

O website foi submetido à avaliação por três categorias de avaliadores: os enfermeiros especialistas nas áreas de informática, os enfermeiros especialistas em emergência e cuidados intensivos, pois estes contemplam as áreas temáticas envolvidas no website, e os enfermeiros generalistas que haviam concluído a graduação no segundo semestre do ano de 2002, num total de 21 avaliadores. $\mathrm{O}$ primeiro grupo atentou, prioritariamente, para a qualidade do conteúdo disponibilizado no site, enquanto o segundo grupo avaliou os recursos computacionais do website, ou seja, a navegação, a utilização de botões para a navegação, o uso de imagens e de cores e o projeto global; o grupo de especialistas contou com a participação de 12 enfermeiros, sendo dois especialistas em informática e dez especialistas clínicos (emergência e cuidados intensivos).

$\mathrm{O}$ intuito da participação dos enfermeiros generalistas (recém-graduados) na avaliação do website foi verificar a utilidade e clareza do material, frente aos conteúdos adquiridos nas disciplinas que compõem o currículo do curso de graduação em enfermagem; desta avaliação, participaram nove enfermeiros.

A pesquisadora acompanhou a visitação do website tanto dos especialistas quanto dos enfermeiros. O tempo utilizado pelo grupo de especialistas e pelo grupo de enfermeiros para a navegação no site foi, em média, de 37 e 21 minutos, respectivamente.

Após a etapa de navegação no website, os avaliadores responderam um questionário de avaliação. É importante mencionar que foram elaborados questionários de avaliação de acordo com os objetivos propostos para cada grupo de avaliadores. Todos os questionários continham perguntas fechadas, nas quais seria atribuído valores que variavam de ruim, bom, muito bom e excelente. Para ambas as categorias de avaliadores haviam duas perguntas abertas destinadas a exposição de opiniões e sugestões à cerca do material visualizado.

Para o grupo de avaliadores classificados como especialistas clínicos o questionário continha onze perguntas. As nove perguntas fechadas tinham como itens: a 
adequação de conteúdo (clareza e objetividade do conteúdo); metodologia utilizada e a relação direta com o entendimento do leitor; a utilização em ambientes de ensino; o uso de cores e o uso de imagens para despertar o interesse e não exaurir o usuário; o manuseio e o "design" do website; a qualidade de informações, incluindo aspectos de veracidade e fidedignidade dos conteúdos e, por fim, o protocolo de condutas apresentado, por oferecer recomendações a esta tarefa e por ser a prática baseada em evidências uma atividade inovadora na enfermagem.

Para o grupo de avaliadores classificados como especialistas em informática, as nove perguntas fechadas abordavam: a utilização dos botões de navegação para que a visitação fosse agradável, sem dúvidas e com várias opções de caminhos dentro do site o tamanho da letra; o uso de imagens; o uso das cores do site; o tempo de troca de telas; se a relação de texto e as imagens eram pertinentes, independente do conhecimento do usuário sobre o assunto; entendimento do texto, ou seja, a gramática adequada sem gerar dúvidas; a quantidade de informações e as características da interface possuindo ambiente agradável com boa estruturação.

Para o grupo de avaliadores classificados como enfermeiros generalistas haviam oito perguntas fechadas no questionário que relacionavam com a clareza e a objetividade do conteúdo, o uso das cores, os vocabulários utilizados, as imagens (as fotos e o vídeo existente no website), a interface e a navegação.

As perguntas abertas que compunham os questionários dos grupos de avaliadores especialistas e generalistas eram destinadas às opiniões e sugestões quanto ao material avaliado.

Com relação às opiniões, de modo geral, os avaliadores, independentemente da categoria, afirmaram que o website é de extrema relevância, de caráter informativo, com excelente conteúdo, de fácil manuseio e que contribuirá para a disseminação de informações e para programas de treinamento e aperfeiçoamento.

A escassez de material bibliográfico brasileiro existente para ser utilizado como fonte de referência à sustentação da prática realizada diariamente pelos profissionais no transporte intra-hospitalar de pacientes graves, é fator determinante para o surgimento do site e para a prestação da assistência a estes indivíduos. Ao fazer uso dos recursos tecnológicos e da velocidade que a informação transita por estes, os usuários com os mais diversos propósitos poderão usufruir dos conteúdos oferecidos e aplicá-los conforme as necessidades.

As sugestões dadas sobre o material avaliado foram relacionadas quanto a correções textuais e ortográficas, modificações de imagens e botões de navegação e ações pertinentes quanto ao bloqueio de imagens e vídeo, a fim de evitar o uso indevido por outros internautas. Foi sugerida, também, a criação de um espaço para discussão virtual e compartilhamento de materiais pertinentes ao assunto. Tais sugestões foram incorporadas no website e o espaço para a discussão virtual será estabelecido futuramente.

\section{O protocolo de condutas}

A Prática Baseada em Evidências (PBE) é um movimento voltado para a formação de profissionais de saúde imbuídos de alto teor de espírito crítico e aptidão para manter o processo de educação continuada; tem contribuído para traçar novas estratégias e métodos didático-pedagógicos e para divulgar outros previamente desenvolvidos $^{(12)}$.

Para a consistência da PBE são necessárias a integração das evidências, da experiência vivenciada na prática, da competência (a tríade conhecimentos, habilidades e atitudes) e da ética. A associação destes itens permite o processo de tomada de decisão pautado na melhor qualidade de assistência ao paciente. Certamente, o que se espera obter é a redução das complicações, dos índices de morbimortalidade e a diminuição de custos.

À medida que as informações são decodificadas, elas são transformadas em guias de conduta (também chamados de guidelines) ou protocolos clínicos (ou diretrizes clínicas). Simplificadamente, guias de condutas referem-se a linhas de conduta, procedimentos a serem adotados, e protocolos clínicos, a orientações e comportamentos sugeridos.

A proposta da idealização de um protocolo de condutas surgiu da constatação da escassez de material bibliográfico no país sobre o tema em questão; também busca oferecer uma forma de padronização para as ações que compõem um tratamento/atendimento, baseado no melhor e mais eficiente conhecimento e prática.

Para que se possa prevenir efeitos adversos no transporte intra-hospitalar, os guias de condutas e ou protocolos devem ser seguidos a fim de se organizar o procedimento $^{(13)}$. Os profissionais devem ser qualificados e os equipamentos necessários para a monitorização clínica do paciente devem estar à disposição para a realização e a excelência do atendimento.

As recomendações sobre o transporte intra-hospitalar foram construídas a partir da pesquisa em fontes de informação baseadas em evidências sobre o assunto e da experiência vivenciada na prática assistencial. O protocolo de condutas foi desenvolvido em três eixos na prestação da assistência ao paciente crítico adulto na ocasião do transporte, seja qual for a indicação terapêutica: avaliação do paciente, avaliação da equipe e avaliação do material/equipamento.

O primeiro eixo está vinculado ao conhecimento e avaliação das condições clínicas do cliente, assim como a monitorização cardiorrespiratória antes, durante e de- 
pois do procedimento. Convém ressaltar que a avaliação do risco/benefício do transporte deve ser levada em consideração, pois determina o sucesso ou o fracasso do procedimento.

O segundo eixo diz respeito à composição, conhecimento cientifico e treinamento da equipe multiprofissional que realizará o transporte intra-hospitalar. A formação da equipe conta com a participação de enfermeiros, médicos, fisioterapeutas e auxiliares de enfermagem. Estes profissionais, dependendo da necessidade do paciente $\mathrm{e}$ da realidade da instituição de saúde, devem ser qualificados, atualizados e a divisão e organização das tarefas durante todas as fases do transporte deve ser preconizada, para garantir a redução ou ausência de complicações para o paciente.

O terceiro eixo está relacionado à avaliação dos equipamentos e dos materiais que fazem parte do transporte. Estes aparatos devem ser checados antes de qualquer procedimento e devem estar em perfeitas condições de uso. Recomenda-se que as manutenções sejam efetuadas rotineiramente.

Cada item contido em cada eixo apresenta um nível de recomendação (NR) e graus de evidência (GR) segundo as determinações do Jornal da Associação Médica Americana ${ }^{(14)}$. Vale ressaltar que o material desenvolvido deve ser considerado apenas como uma recomendação em decorrência de sua não validação (Figura 2).

Para a avaliação do material desenvolvido, contouse com a participação de uma enfermeira especialista em prática baseada em evidências. Esta profissional apontou algumas modificações e as correções foram efetuadas de acordo com as orientações recebidas.

$\mathrm{Na}$ ocasião da avaliação do website, as recomendações contidas neste protocolo também foram submetidas a este processo. Verificou-se que os especialistas em cuidados intensivos e emergência consideraram pertinente sua confecção e sua aplicação no processo de cuidar.

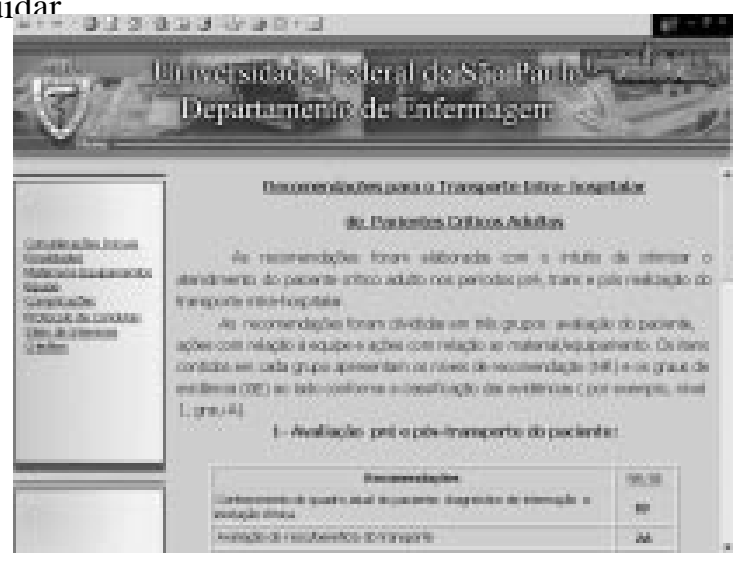

FIGURA 2 - Recomendações sobre transporte Intra-hospitalar de Pacientes Críticos Adultos, SP, 2003.

\section{CONCLUSÕES}

O site intitulado como Informações On-line sobre Transporte Intra-hospitalar de Pacientes Críticos Adultos, desenvolvido durante o Programa de Pós-Graduação em Enfermagem da Universidade Federal de São Paulo, está disponível na rede mundial de computadores, após a avaliação e aprovação por 12 especialistas nas áreas de informática, emergência e cuidados intensivos e por nove enfermeiros generalistas. Neste endereço também é encontrado o protocolo de condutas para transporte intra-hospitalar de pacientes críticos adultos, desenvolvido na mesma ocasião do website.

A atualização periódica do material, substituição de imagens, inclusão de contador de acesso e correio eletrônico são ações idealizadas no processo de manutenção e avaliação do website. A criação de um grupo de discussão virtual e a expansão das informações sobre o assunto a quem possa interessar, também são propostas pretendidas.

A aglutinação destes recursos é a nova estratégia de mudança de comportamento que a educação e a saúde necessitam, esperando que usuários e especialistas façam o melhor proveito desta informação, em busca de suas aspirações pessoais e profissionais.

\section{REFERÊNCIAS}

1. Moran JM. O que é educação à distância [texto na Internet]. 2000 [citado 2002 Abr 08]. Disponível em: http://www. eca. usp.br/prof/moran/textost.htm.

2. Forman D, Nyatanga L, Rich T. E-learning and education diversity. Nurse Educ Today. 2002;22(1):76-82.

3. Diogo RCS. Desenvolvendo um website educacional sobre intervenção de enfermagem: aspiração de secreções traqueobrônquicas [tese]. São Paulo (SP): Departamento de Enfermagem, Universidade Federal de São Paulo; 2001.

4. Marin HF. Vocabulário: recurso para a construção de dados em enfermagem. Acta Paul Enferm. 2000;13(1):86-9.

5. Trochim WMK. Evaluating websites [text in the Internet]. 1999 [cited 2002 Jun 17]. Available from: http://www.trochim. human. cornel.edu/webval/ webintro/webintro.htm.)

6. Motta MCS. Software de enfermagem em saúde do lactente: desenvolvimento e validação [tese]. Rio de Janeiro (RJ): Universidade Federal do Rio de Janeiro; 2001.

7. Salvador E. Multimídia e internet [texto na Internet]. 2000. [citado 2002 Abr 12]. Disponível em: http://www.virtual. epm.br/material/tis 
8. Agency For Health Care Policy And Research. Assessing the quality of internet health information. Summary. [text in the Internet] 2001. Rockville, MD, and Mitretek Systems, McLean, VA. [cited 2002 Jul 31] Available from: http://www.ahrq.gov/ data/infoqual.htm

9. Health on the Net Foundation. Código de Conduta (HONcode) para sites Web medicina e saúde [texto na Internet]. [citado 2000 Jul 31] Disponível em: http://www.hon.ch/HONcode/ Portuguese/index.html

10. Conselho Federal de Enfermagem. Resolução n. 274/2002. Dispõe sobre a utilização da Internet, pelos profissionais de enfermagem e dá outras providências [texto na Internet]. [citado 2003 Jan 07] Disponível em: http://www.corensp.org.br/ resolucoes/resolucoes.htm
11. Beretta MIR; Andrade AS. Avaliação de um curso de extensão universitária. Acta Paul Enferm. 2000;13(1):92-100.

12. Lopes AA. Medicina baseada em evidências: a arte de aplicar o conhecimento científico na prática clínica. Rev Assoc Med Bras. 2000;46(2):285-8.

13. Waydhas C. Intrahospital transport of critically ill patients. Engl Crit Care. 1999;3(1):83-9.

14. Guyatt GH, Haynes RB, Jaeschke RZ, Cook DJ, Green L, Naylor CD, et al. Users' Guides to the Medical Literature: XXV. Evidence-based medicine: principles for appyi the Users' Guides to patient care. JAMA. 2000;284(10):1290-6. 